Research Article

www.jestr.org

\title{
A New Sharpening Technique for Medical Images using Wavelets and Image Fusion
}

\author{
P. Zafeiridis ${ }^{1,}$, N. Papamarkos ${ }^{1}$, S. Goumas ${ }^{2 . *}$ and I. Seimenis ${ }^{3}$ \\ ${ }^{1}$ Department of Electrical \& Computer Engineering, Democritus University of Thrace, GREECE \\ ${ }^{2}$ Information Management Department, Technological Educational Institute of Kavala, GREECE \\ ${ }^{3}$ Department of Medicine, Democritus University of Thrace, GREECE
}

Received 3 MAy 2016; Accepted 20 July 2016

\begin{abstract}
In this work a new image sharpening technique based on multiscale analysis and wavelet fusion is presented. The proposed technique is suitable for visibility optimization of biomedical images obtained from MRI sensors. The proposed approach combines, with a wavelet based fusion algorithm, the sharpening results accrued from a number of independent image sharpening techniques. Initially, the input image is preprocessed by a denoising filter based on a complex Two Dimensional Dual-Tree Discrete Wavelet Transform. Then, the denoised image is passed through a cluster of five sharpening filters and subsequently, the final image is obtained with the help of a wavelet fusion technique. The main novelty of the proposed technique lies on using only one input image for sharpening and that the fusion is performed on images extracted in different frequency bands. This technique could be used as a preprocessing step in many applications. In this paper we focus on the application of the proposed technique in brain MR images. Specific image sharpening and quality indices are employed for the quantitative assessment of the proposed technique.
\end{abstract}

Keywords: Image sharpening, wavelets, image fusion, image quality assessment

\section{Introduction}

In general, image sharpening belongs to a category of image processing techniques called spatial filtering and it deals with enhancing details information in images. The details information is typically contained in the high frequency components of an image and corresponds to spatial regions that include edges and other small image features. Thus, most of the image sharpening techniques tries to enrich the high frequency contents of the image by using a high pass filtering. Specifically, according to this approach, we add to the original image a signal that is proportional to a high-pass filtered version of the original image. This enhancement of the high-frequency components of an image leads to an improvement in the visual quality.

The incentive of this work comes from multiscaling techniques and multiresolution analysis used in image fusion. As an important application, it can be used for suitably sharpening of medical images by using multiscale analysis and multimodal fusion. It should be noted that image sharpening using fusion is usually performed when we have more than one version of the same image scene. However, this is not always possible because in most of the cases, we want to emphasize the image details while only one image is available. In the proposed technique the sharpening is performed by using only one image, e.g. one frame from magnetic resonance imaging (MRI) sensors. This particular kind of images was selected due to its special

*E-mail address: goumas@teiemt.gr ISSN: 1791-2377 (C) 2016 Eastern Macedonia and Thrace Institute of Technology. All rights reserved. features i.e. large homogenous areas with high contrast. These features give poor sharpening results when classic sharpening filters like unsharp masking or other linear filters are applied to these images. The final image is obtained by a fusion procedure applied on different frequency bands of the image. Specifically, the key idea is to apply five different, sharpening filters (concerning different frequency bands) to the initial image and then combine the resulting images to one image through fusion. This approach highlights the numerous subtle details in different frequency bands and produces a suitable sharpened image. The two dimensional Discrete Wavelet Transform (2D DWT) and the two dimensional Stationary Wavelet Transform (2D SWT) approaches are used for this fusion scheme.

Wavelet transform plays an important role in image processing [1-2]. DWT [2-3] is one of the recent wavelet transforms used in image processing. 2D DWT decomposes an image into different subband images [4-5], namely lowlow (LL), low-high (LH), high-low (HL), and high-high $(\mathrm{HH})$. SWT is another recent wavelet transform used in several image processing applications. In short, SWT is similar to DWT but it does not use down-sampling, hence the subbands will have the same size as the input image. Input and subband images obtained by DWT are interpolated [6] with a factor of $\alpha / 2$, where $\alpha$ denotes the number of decompositions [7-8].

Image fusion can be broadly defined as the process of combining multiple input images of the same scene or some of their features into a single image without the introduction of distortion or loss of information [9-10]. The aim of image fusion is to integrate complementary as well as redundant information from multiple images to create a fused image 
output. The result of image fusion is a new image which is more suitable for human visual and machine perception or further image processing and analysis tasks such as segmentation, feature extraction and object recognition [1011]. The successful fusion of images acquired from different modalities or instruments is of great importance in many applications, such as medical imaging, remote sensing, computer vision, robotics, defense surveillance, nondestructive evaluation and microscopic imaging [12-14].

According to the stage at which the combination mechanism takes place, the image fusion methods can be generally grouped into three categories, namely, pixel level, feature level and symbol level or decision level [10], [1213]. Image fusion at pixel level means fusion at the lowest processing level referring to the merging of measured physical parameters [13]. It generates a fused image in which each pixel is determined from a set of pixels in the various sources. Feature level fusion deals with the fusion of features such as edges, size, and shape and texture. Fusion at symbol level allows the information to be effectively combined at the highest level of abstraction. Since the pixel level fusion has the advantage that the images used contain the original measured quantities, and the algorithms are computationally efficient and easy to implement, the most image fusion applications employ pixel level based methods [10], [13]. There are various methods that have been developed to perform image fusion. Some well-known image fusion methods are referred analytically in [4]. The selection of the appropriate one depends on the type of application. In particular, some commonly used techniques in pixel level fusion are: weight combination, optimization approach [8], [15-16], principal component analysis (PCA) [4], multiresolution (MR) decompositions [13-14] and neural networks [17-18]. Some generic requirements can be imposed on the fusion result [13-14]: (a) the fused image should preserve as closely as possible all relevant information contained in the input images; (b) the fusion process should not introduce any artifacts or inconsistencies, which can distract or mislead the human observer, or any subsequent image processing steps; and (c) in the fused image, irrelevant features and noise should be suppressed to a maximum extent.

Medical image fusion is based on information fusion and image fusion. The fusion in medical images can often lead to additional clinical information not apparent in the separate images [10-19]. Multimodal medical image fusion has emerged as a promising new research area in recent years. Using medical images from multiple modalities, such as $\mathrm{X}$ ray, computed tomography (CT), magnetic resonance imaging (MRI), and positron emission tomography (PET) images, increases robustness and enhances accuracy in biomedical research and clinical diagnosis [20-21]. Another advantage is that the fusion of medical images can reduce the storage cost by storing just the single fused image instead of multisource images [10].

Image sharpening is directly related with the image quality. In most of the cases the application of image sharpening suitably increases and improves the image quality. Measurement of image quality is a challenging problem in many image processing fields from image sharpening to image fusion [22]. In the literature, the image quality evaluation methods can be classified into subjective (or qualitative analysis) and objective methods (or quantitative analysis) [22], [27]. Subjective methods are based on human judgment and operate without reference to explicit criteria [22]. The subjective quality evaluation method depends on the expert's experience and some uncertainty is involved because this measure has no rigorous mathematical models and is mainly visual. Objective methods are based on comparisons using explicit numerical criteria and several references are possible such as the ground truth or prior knowledge expressed in terms of statistical parameters and tests. The objective image quality measures are mathematically defined measures such as peak signal to noise ratio (PSNR), root mean squared error (RMSE) and image entropy [28-31]. Comparative analysis of image fusion methods demonstrates that different metrics support different user needs, sensitive to different image fusion methods, and need to be tailored to the application [25]. Categories of image fusion metrics are based on information theory, features, structural similarity, or human perception [32]. Spatial frequency [23] that measures the activity level of an image is a suitable index for evaluating the quality of fused images. Finally, Piella and Heijmans [26] proposed a quality index for image fusion based upon the Wang - Bovik (2002) Universal Quality Index (UQI) [24].

In this paper, a novel sharpening method is proposed based on multiscale analysis and wavelet fusion. The approach is implemented as a three-stage procedure. The first is a preprocessing stage where we perform image denoising based on a complex 2D DWT [33-34]. This procedure removes high frequency noise without affecting the important image details. In the second stage, in order to exploit important image details, the image is passed through a cluster of sharpening filters. Most of these filters embed Gaussian filtering that allows us to exploit image information at different scales. Accordingly, the stage can be considered as a multiscale approach that emphasizes image information at different frequency subbands of the image. Specifically, in the second stage we use five different image sharpening techniques, namely unsharp masking, local contrast enhancement, various mask sharpening filters, maxmin linear sharpening filter and contrast-limited adaptive histogram equalization (CLAHE) [43]. Each sharpening method has several parameters which were tuned for best results, according to two indexes that are associated with the amount of image sharpening, i.e. spatial frequency and entropy. Next, in the third stage, the images obtained from the previous stage are fused with the use of 2D DWT and 2D SWT resulting in a final, sharpened image. It should be noted that the main novelty of the proposed technique is focused on the use of only one input image and that the fusion is performed only on images extracted in different frequency bands. The method was tested for MR brain images and was evaluated with the two aforementioned sharpening measures and with the Piella metric for fusion. Finally, it was compared with two new sharpening techniques proposed by Deng [35] and Ying [36] respectively.

The remaining of the paper is organized as follows. Section 2 describes the method and its aspects. In section 3 the experimental results are presented along with the performance evaluation. Finally, section 4 concludes the present work.

\section{Description of the Sharpening Technique}

The proposed technique for image sharpening is depicted in Fig.1. Initially, a denoising filter that uses Discrete Wavelet Transform (DWT) is applied to an input image as a 
preprocessing step. Then, five different spatial filters for image sharpening are applied, in parallel, to the denoised image. These filters are based on the known sharpening techniques unsharp masking [29], Local Contrast enhancement [37], various Mask Sharpening Filters [29], Max-Min Linear sharpening filter [38], and Contrast-limited Adaptive Histogram Equalization (CLAHE) [39].

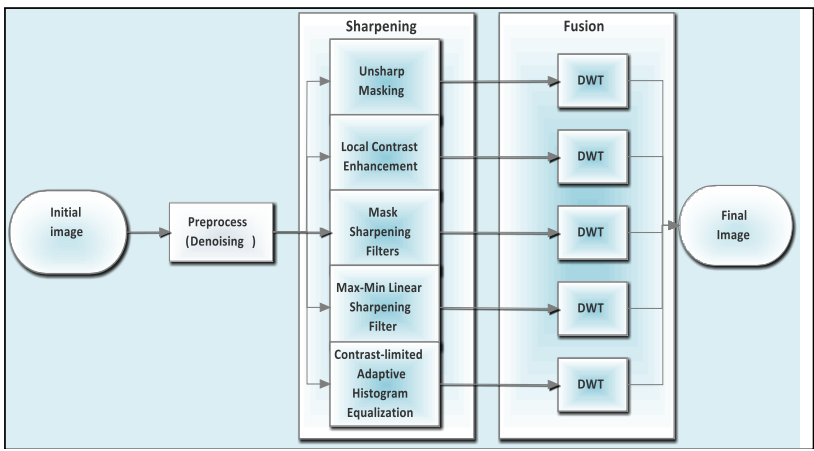

Fig.1. Block diagram of proposed sharpening technique

The mentioned techniques were tested for various values of their parameters when applied to a specific image. As it is mentioned above, the performance evaluation of the resulted sharpened images was based on two sharpening metrics: spatial frequency and entropy. The optimal image, according to the two metrics employed, for each technique, was selected to proceed in the next step of the process which attains image sharpening through image fusion. The fusion was achieved with the use of 2D DWT [40]. DWT filter with specific wavelet function and multiresolutional analysis level was applied to each image that resulted from the application of the sharpening filters mentioned above. Subsequently, fusion of coefficients with various coefficient selection methods (specifically, 3 different methods were tested) was applied. The final fused image resulted by applying the 2D Inverse Wavelet Transform in the concluded array with the fused coefficients. The quality of the fused image was evaluated with the two aforementioned metrics as well as by comparing between the input and the fused images according to Piella and Heijmans image fusion quality measure [26]. A detailed description of the proposed method's steps is provided in the following paragraphs.

\subsection{Pre-processing (Denoising Method)}

As a pre-processing step, a denoising method based on wavelet analysis was used. Specifically, the denoising technique used is wavelet thresholding (or "shrinkage") [3334]. When we decompose data using the wavelet transform, we use filters that act as averaging filters, and others that produce details. Some of the resulting wavelet coefficients correspond to details in the data set (high frequency subbands). If the details are insignificant, they might be omitted without substantially affecting the main features of the data set. The idea of thresholding is to set all high frequency subband coefficients that are less than a particular threshold to zero. These coefficients are used in an inverse wavelet transformation to reconstruct the data set. Using complex 2D dual-tree DWT implemented on the original ("noisy") image and a threshold value of about 0.015 , (in normalized values of grayscale images) gave the best results. In this instance and in experimental results presented in Section 3, subjective image quality analysis was performed by one reader blinded to image parameters. Image quality assessment employed a 3-point grading system (good, moderate and poor quality) based on image sharpness, clarity of grey-white matter interface and presence/absence of artifacts. An example of application of this denoising method on a T1-weighted MR image of the brain is presented in Fig. 2.

$$
\operatorname{PSNR}\left(\frac{\text { Denoised Image }}{\text { Original Image }}\right)=51.035
$$

$\mathrm{h}$ as possible the noise of input image. This can be accomplished with the use of, i.e. a Gauss filter.

Step 2. Image $I_{s}$ is subtracted from input image $I$ and the resulting image is $I_{\text {mask }}$

$$
I_{\text {mask }}=I-I_{S}
$$

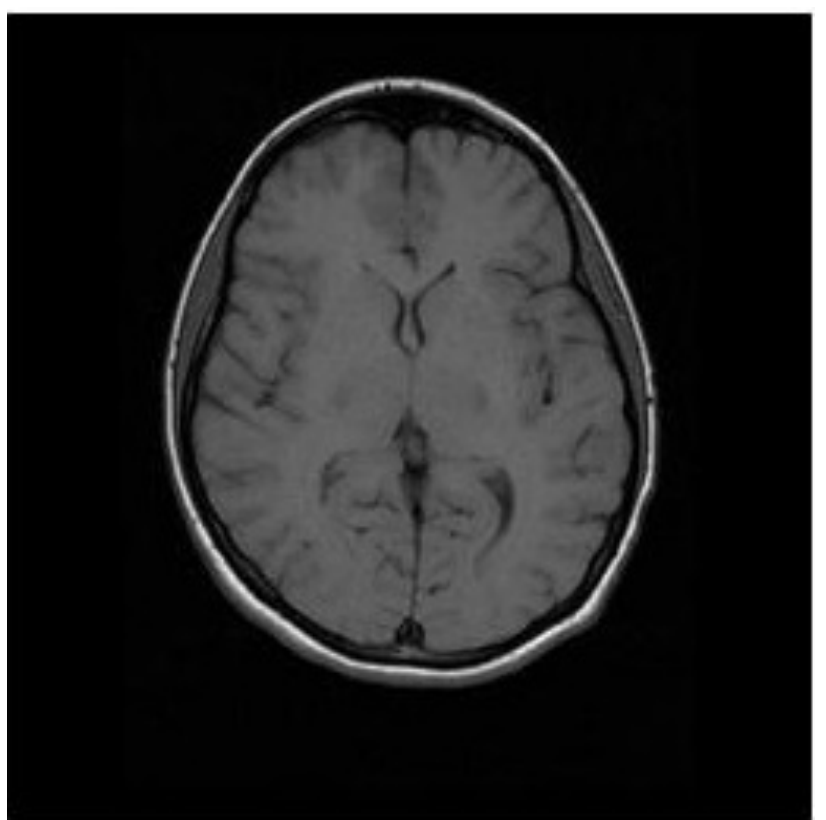

a)

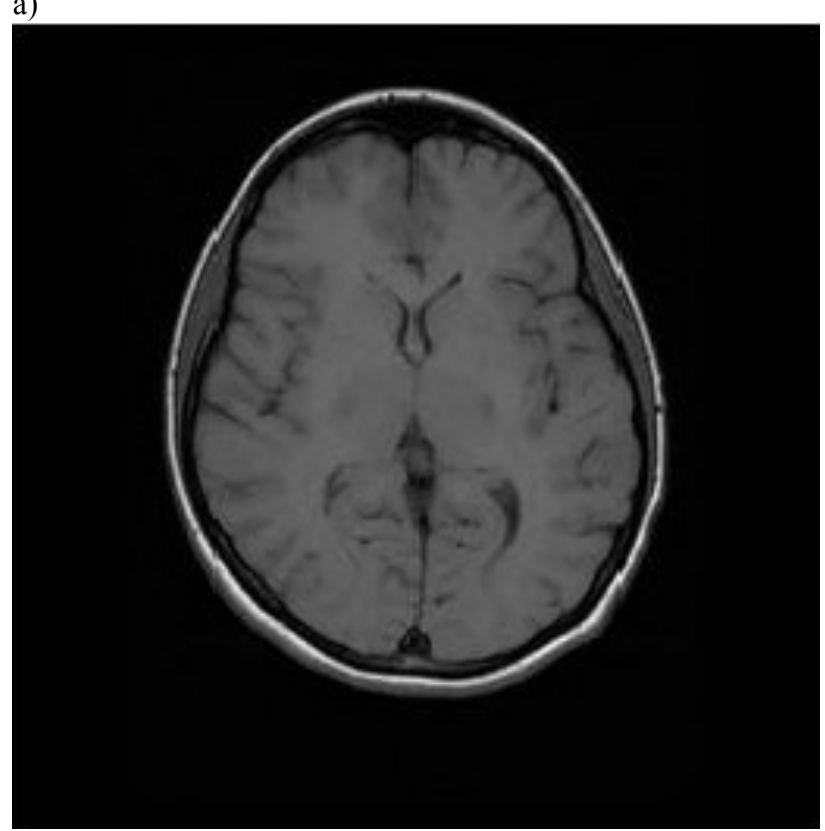

b)

Fig. 2. (a) Original MRI brain Image and (b) Denoised MR brain Image 


\subsection{Sharpening filters used}

\subsubsection{Unsharp masking}

The first sharpening filter used is Unsharp masking. The technique is based in the subtraction of a blurred (un-sharp) version of the image from the original one. It allows the mixture of low and high frequencies of an image. Depending on a factor that controls this mixture it can either result in a blurred image or a sharpened image. The main steps of the method are:

Step 1. A smoothed version $I_{S}$ of input image $I$ is derived. This process must preserve most of details of the image while reducing as muc

Image $I_{\text {mask }}$ is characterized as mask that is substantially a version of the initial image with high frequency content. Thus, image $I_{\text {mask }}$ can be calculated also with the use of a high pass filter.

Step 3. The output image is calculated using a formula as below:

$$
I_{\text {unsharp }}=I+k I_{\text {mask }}=I_{S}+(k+1) I_{\text {mask }}
$$

with $k>0$. The reasonable value for $k$ varies from 0.2 to 1.0 with the larger values providing increasing amount of sharpening. If $k>1$ then we have the case of a high boost filtering.

Fig. 3 depicts an example of the application of unsharp masking in an MR image.

\subsubsection{Local Contrast Enhancement}

The second sharpening technique is Local Contrast Enhancement. The contrast increases in specific areas and not in the entire image. Using this technique makes it possible to preserve the intensity in homogeneous areas of the image and also in areas with high contrast. The rest of the pixels change their intensity value according to a specific transformation in order to increase contrast. This adaptive way of contrast enhancement can be described in the following steps [41]:

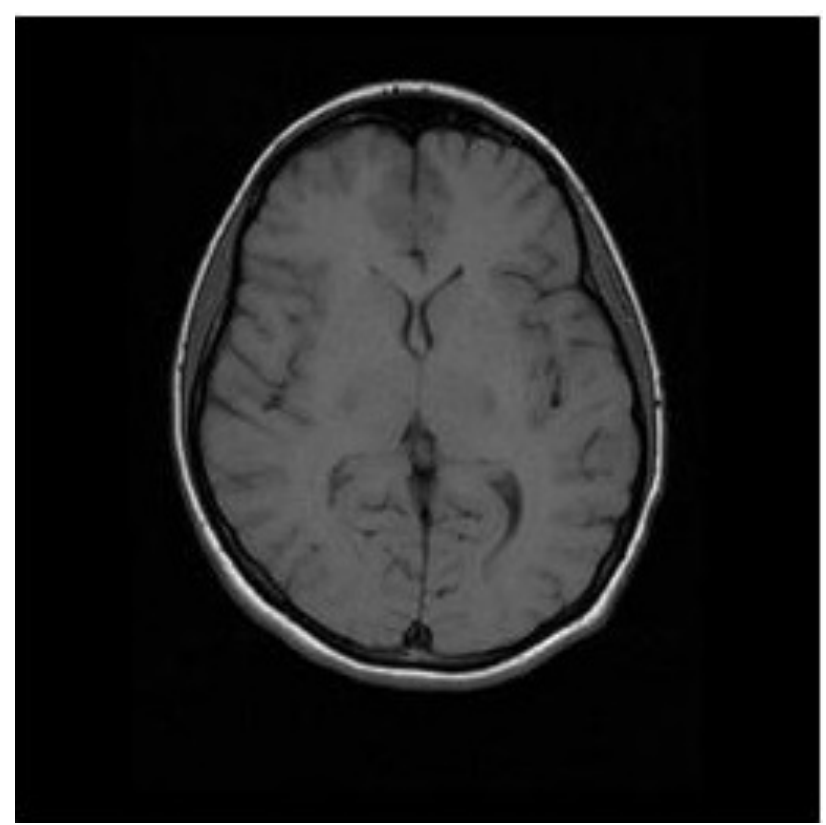

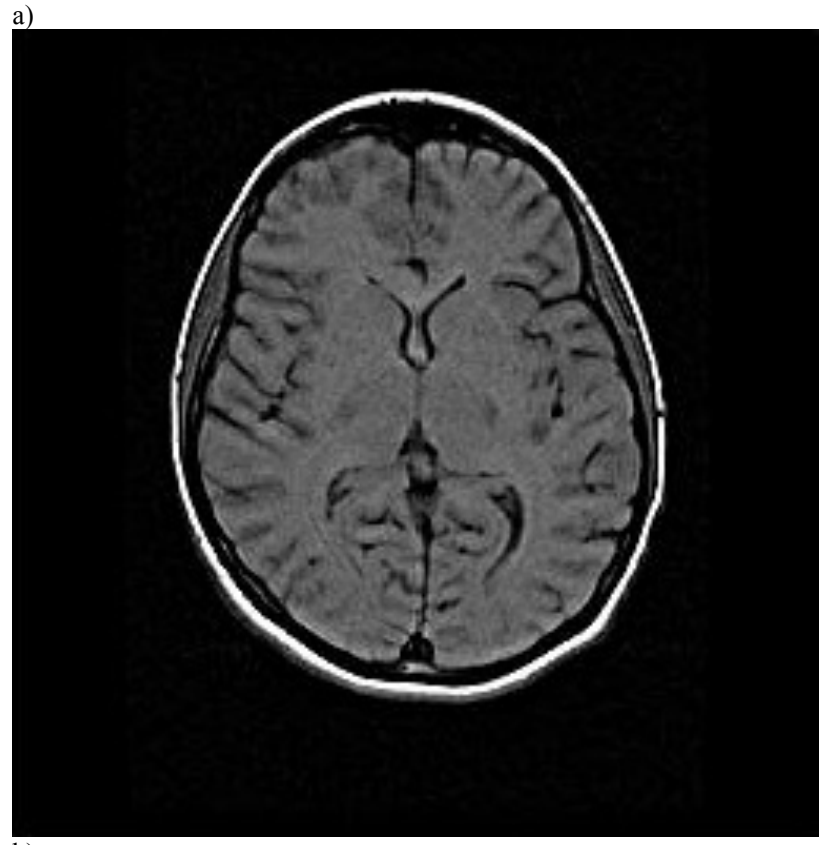

b)

Fig. 3. (a) Original MR brain Image and (b) Unsharped MR brain Image

\subsubsection{Local Contrast Enhancement}

The second sharpening technique is Local Contrast Enhancement. The contrast increases in specific areas and not in the entire image. Using this technique makes it possible to preserve the intensity in homogeneous areas of the image and also in areas with high contrast. The rest of the pixels change their intensity value according to a specific transformation in order to increase contrast. This adaptive way of contrast enhancement can be described in the following steps [41]:

Step 1: If $N$ is a neighbourhood of pixels (the usual case is a $3 \times 3$ window), then the mean contrast of the central pixel in place with coordinates $(n, m)$ can be calculated as in eq. (2.3)

$$
\bar{C}(n, m)=\frac{1}{8} \sum_{\substack{i=-1 \\(i . j) \neq(0,0)}}^{1} \sum_{j=-1}^{1} \frac{|I(n, m)-I(n+i, m+j)|}{I(n, m)+I(\mathrm{n}+\mathrm{i}, \mathrm{m}+\mathrm{j})}
$$

Step 2: If $T_{1}$ and $T_{2}$ are two threshold values in the interval $[0,1]$, then the contrast or, consequently the brightness of pixel $(n, m)$ will change its value if only $T_{1} \leq \bar{C}(n, m) \leq T_{2}$. Specifically, a pixel will change its value according to the next rule:

$$
I(n, m)=\left\{\begin{array}{l}
T(I(n, m)), \text { if } T_{1} \leq \bar{C}(n, m) \leq T_{2} \\
I(n, m), \text { else }
\end{array}\right.
$$

where $T(g)$, denotes a histogram transformation function that can be chosen in relation with the histogram form of an image. The function used in this case is 


$$
T(g)=\left\{\begin{array}{l}
0, \text { if } \mathrm{g} \leq \mathrm{x}_{1} \\
\operatorname{round}\left(y_{1}+\frac{\left(y_{2}-y_{1}\right)\left(g-x_{1}\right)}{x_{2}-x_{1}}\right), \text { if } x_{1}<g<x_{2} \\
1, \text { if } g \geq x_{2}
\end{array}\right.
$$

where $\left(x_{1}, y_{1}\right)=(0.3,0)$ and $\left(x_{2}, y_{2}\right)=(0.7,1)$ are two points in the (normalized) histogram that define the straight line of the transformation function. The threshold values used are $T_{1}=0$ and $T_{2}$ that varies from 0.1 to 0.9 with step 0.05 . An example of this sharpening technique is presented in Fig. 4.
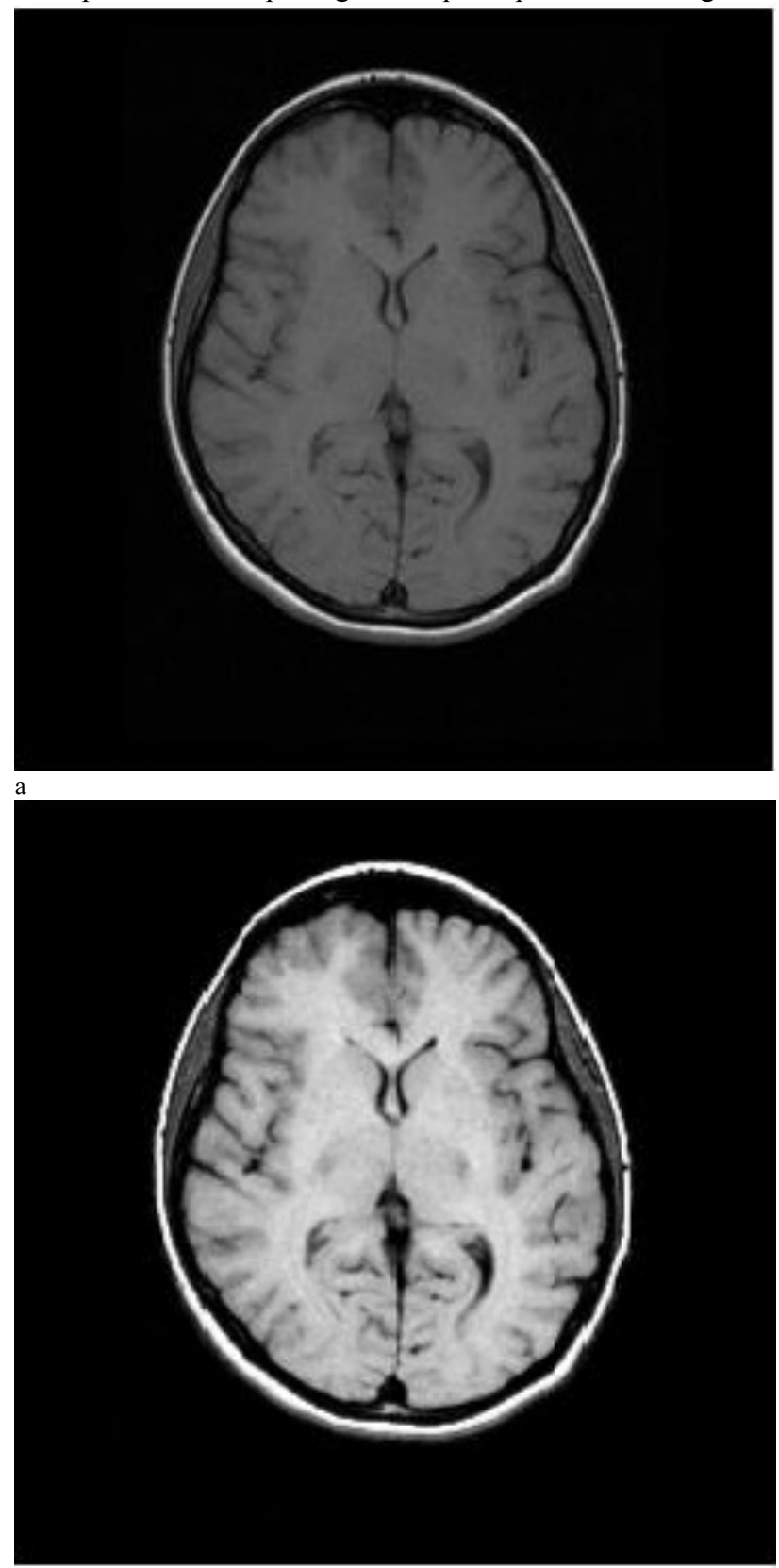

b)

Fig. 4. (a) Original MR brain Image (b) Local Contrast Enhancement MR brain Image

\subsubsection{High-pass filtering}

The third sharpening technique involves high-pass filtering with sharpening masks. The masks are $n x n$ matrices with specific matrix elements. In order to preserve the low frequencies of an image, the central element of the matrix obtains a value that results in one, when all matrix elements are summed. Specifically, if $S$ is the sum of all elements except the central, and the value of the central is $S+K$, then, dividing all elements with $K$ results in the desired matrix. Convolution of an image with this kind of matrix results in an image with sharpened features. In this process there were tested five masks, as shown below.

$$
\begin{aligned}
& \left(\begin{array}{ccc}
0 & -1 & 1 \\
-1 & 5 & -1 \\
0 & -1 & 0
\end{array}\right) \\
& \left(\begin{array}{ccc}
1 & -2 & 1 \\
-2 & 5 & -2 \\
1 & -2 & 1
\end{array}\right) \text {, } \\
& \frac{1}{7}\left(\begin{array}{lll}
-1 & -2 & -1 \\
-2 & 19 & -2 \\
-1 & -2 & -1
\end{array}\right) \text {, } \\
& \frac{1}{14}\left[\begin{array}{rrrcc}
0 & -1 & -1 & -1 & 0 \\
-1 & -2 & -4 & -2 & -1 \\
-1 & -4 & 50 & -4 & -1 \\
-1 & -2 & -4 & -2 & -1 \\
0 & -1 & -1 & -1 & 0
\end{array}\right], \\
& \frac{1}{10}\left[\begin{array}{ccccc}
-1 & -1 & -1 & -1 & -1 \\
-1 & -2 & -2 & -2 & -1 \\
-1 & -2 & 42 & -2 & -1 \\
-1 & -2 & -2 & -2 & -1 \\
-1 & -1 & -1 & -1 & -1
\end{array}\right]
\end{aligned}
$$

Figure 5 presents the result of the convolution of an image with a sharpening mask.

\subsubsection{Max-Min filter}

The fourth sharpening technique that was used is Max-Min filter. It is considered as a recursive, non-linear sharpening filter that can emphasize fuzzy contours or objects [42]. It is based in the pixel intensity comparison between maximum and minimum intensities in a neighborhood. The value of the central pixel is replaced by the nearest extreme pixel value of the neighboring pixels. Specifically, according to Kramer and Bruchner [38], if $I$ is the image, $N(i, j)$ the neighborhood of pixel $(i, j)$ and if

$$
\begin{aligned}
& p_{\text {max }}=\max (N(i, j)) \\
& p_{\text {min }}=\min (N(i, j))
\end{aligned}
$$

then the sharpening transformation is applied according to the following formula:

$$
I^{n+1}(i, j)=\left\{\begin{array}{l}
p_{\max }, \text { if } p_{\max }-I^{n}(i, j) \leq I^{n}(i, j)-p_{\min } \\
p_{\min }, \text { elsewhere }
\end{array}\right.
$$



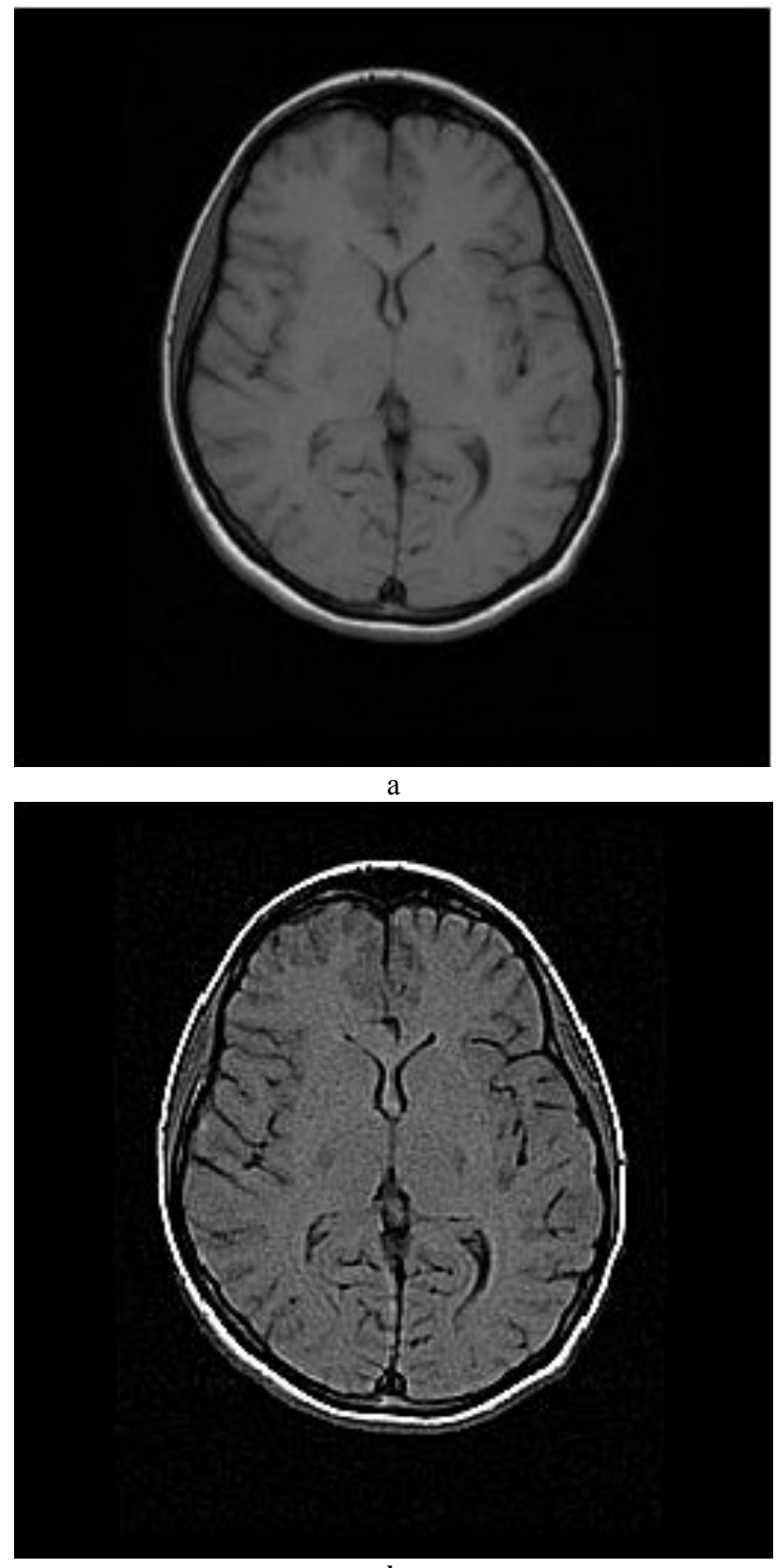

b

Fig. 5. (a) Original MR brain Image (b) MR brain Image after sharpening

where $n=1,2, \ldots$ denotes the number of times that the transformation is applied to the image. This iterative application stops when the result, as observed by a user, is satisfying or until there is not significant change in the result. An example of the application of the Max-Min Transformation is shown in Fig. 6.

\subsubsection{Contrast Limited Adaptive Histogram Equalization}

The last sharpening technique that was used is Contrast Limited Adaptive Histogram Equalization (CLAHE) [43]. CLAHE was developed as an improved version of the wellknown Adaptive Histogram Equalization (AHE). This technique has proven to be successful for medical images contrast enhancement. In classical AHE the image can be considered as a whole but also is divided in a grid of rectangular contextual regions in which the optimal contrast must be found by calculating the histogram of the contained pixels. Calculation of the corresponding cumulative histograms results in a gray-level assignment table that optimizes contrast in each of the contextual regions. The drawback of AHE method is that it enhances also the background noise of an image. This problem can be reduced by limiting the contrast enhancement specifically in homogeneous areas. These areas can be characterized by a high peak in the histogram associated with the contextual regions since many pixels fall inside the same gray range.
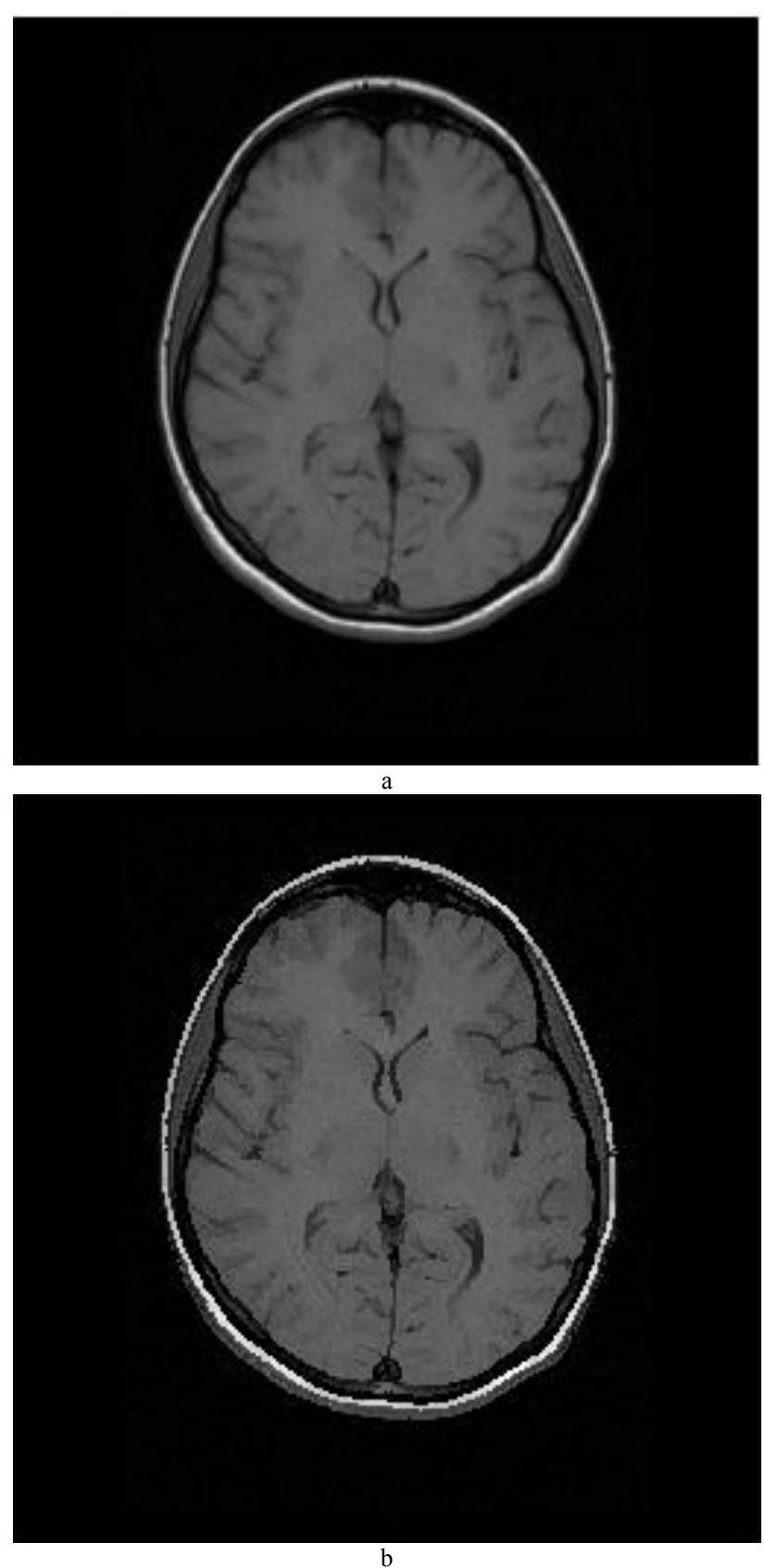

Fig. 6. (a) Original MR brain Image (b) Max-Min sharpening of an MR brain Image

The contrast amplification in the vicinity of a given pixel is obtained by the slope of the transformation function. This is proportional to the slope of the neighborhood cumulative distribution function (CDF). Therefore, CLAHE limits the contrast amplification by clipping the histogram at predefined limits before computing the CDF. This limits the slope of the CDF and therefore of the transformation function. The value at which the histogram is clipped, the so-called clip limit, depends on the normalization of the histogram and thereby on the size of the neighborhood 
region. It is advantageous not to discard the part of the histogram that exceeds the clip limit but to redistribute it equally among all histogram bins, as depicted in Fig. 7.

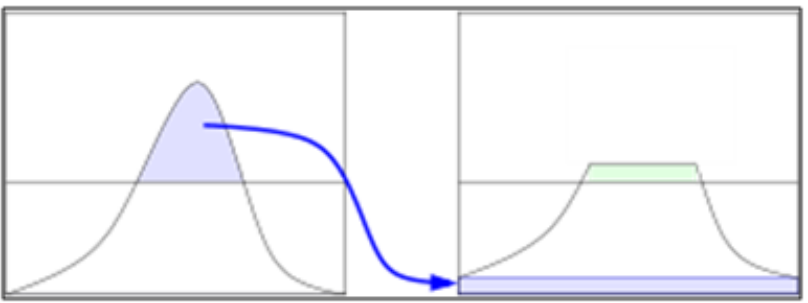

Fig 7. Clip Limitation in Advanced Histogram Equalization

The redistribution will push some bins over the clip limit again (region shaded green in the figure), resulting in an effective clip limit that is larger than the prescribed limit and the exact value of which depends on the image. If this is undesirable, the redistribution procedure can be repeated recursively until the excess is negligible.

The neighboring regions (also called tiles) are then combined using bilinear interpolation to eliminate artificially induced boundaries. The typical size of tiles is $8 \mathrm{X} 8$. This tile size was used in this technique. The Rayleigh Distribution was used for the CDF. The parameters that were tested as variables are the Clip limit, a real number ranging in $[0,1]$, with higher numbers resulting in more contrast and the Alpha parameter, a nonnegative real number that affects the shape of the CDF. An example of the application of this transformation in a gray scale medical image is presented in Fig. 8.

\subsection{Image Fusion Based on Wavelets}

In general, the problem that image fusion tries to solve is to combine multiple input images of the same scene or some of their features into a single image without the introduction of distortion or loss of information [8-9]. As a result, the fused image has better quality than any of the original images and it combines information from different modalities, as long as it concerns medical images.

Wavelet transforms have been successfully used in many fusion schemes. A common wavelet transform technique used for fusion is the 2D DWT [14], [44-45]. It is a spatialfrequency decomposition that provides flexible multiresolution analysis of an image. The one-dimensional (1D) DWT involves successive filtering and down sampling of the signal. Provided that the filters used are bi-orthogonal, they will have a set of related synthesis filters that can be used to perfectly reconstruct the signal. For images the 1D DWT is used in two dimensions by separately filtering and downsampling in the horizontal and vertical directions. This gives four sub-bands at each scale of the transformation with sensitivity to vertical, horizontal and diagonal frequencies.

As it is depicted in Fig. 9, image fusion can be performed by using images of identical or even different resolutions. In our technique, we follow the first approach shown in Fig. 9(a).

The key step in image fusion based on wavelets is that of coefficient combination, namely, the process of merging the coefficients in an appropriate way in order to obtain the best quality in the fused image. This can be achieved by a set of strategies. The simplest is to take the average of the coefficients to be merged, but there are other merging strategies with better performances, such as the selection of the Maximum Absolute Value, the general or adaptive weighted average, region-based fusion schemes and other coefficient combining methods presented in [11].
Figure 10 depicts the procedure of image fusion of many images, of the same resolution. Specifically, in our approach, the input images to be fused were the five images obtained from the sharpening filters presented in Section 2.2. Each of these images was decomposed in various decomposition levels, from level one to five. Two different wavelet functions are selected after testing with the majority of the available various wavelet functions. Finally, two different wavelet transforms were tested: the 2D DWT and the 2D SWT which is also known as "algorithm a trous", algorithm with holes. The SWT [46] is an inherently redundant scheme as the output of each decomposition level contains the same number of samples as the input - so for a decomposition of $\mathrm{N}$ levels there is a redundancy of $\mathrm{N}$ in the wavelet coefficients. This way SWT overcomes the lack of translation-invariance of DWT. The same fusion scheme depicted in Fig. 10 is applied both for 2D DWT and 2D SWT.
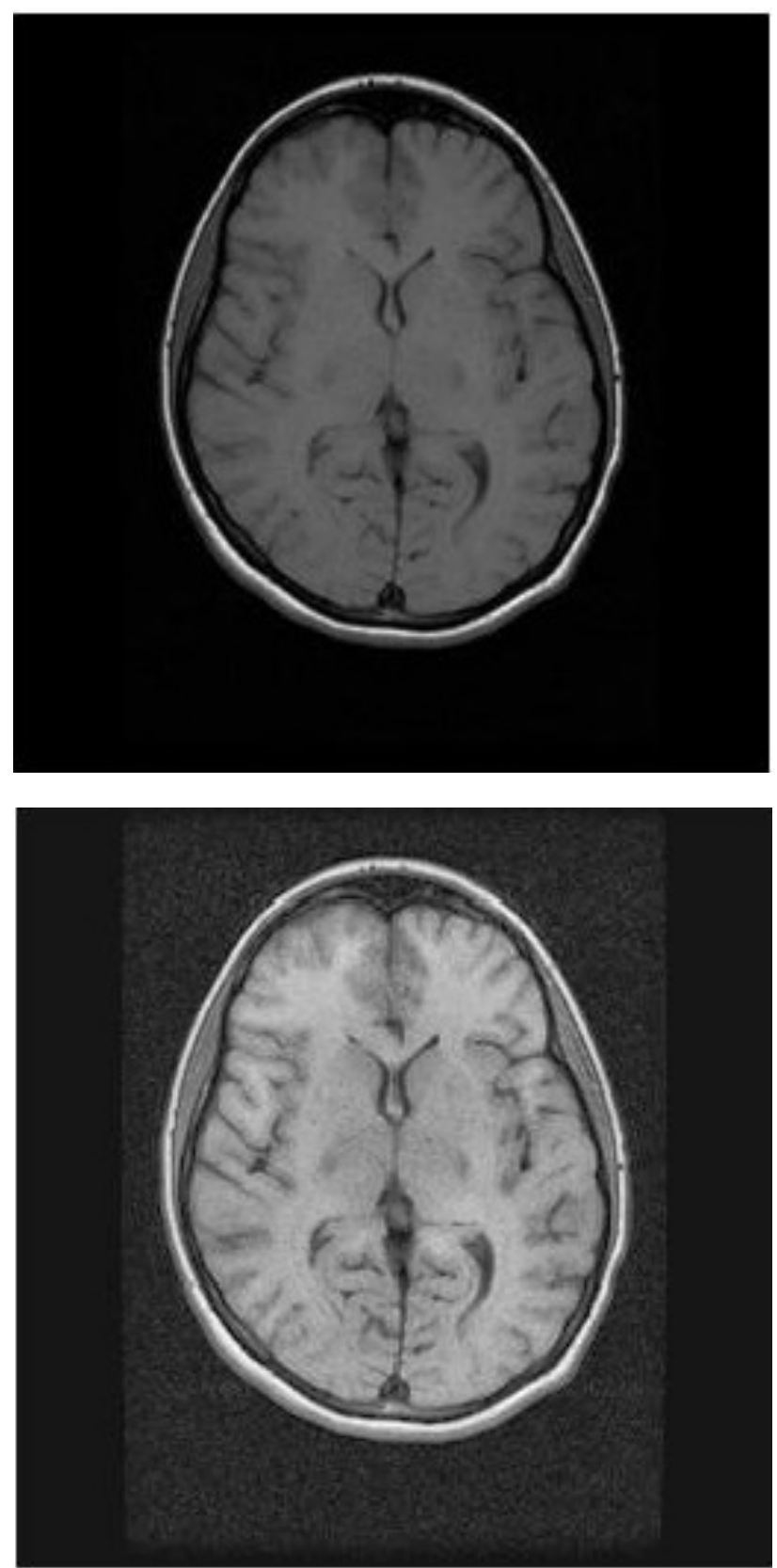

Fig. 8. (a) Original MR brain Image (b) CLAHE sharpened MR brain Image 


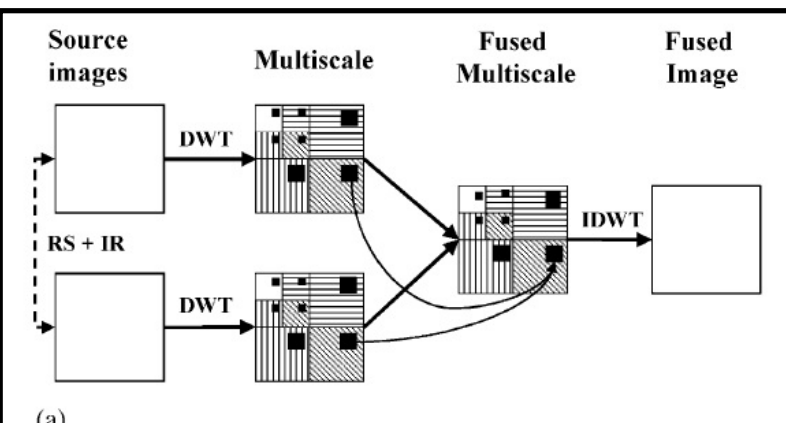

(a)

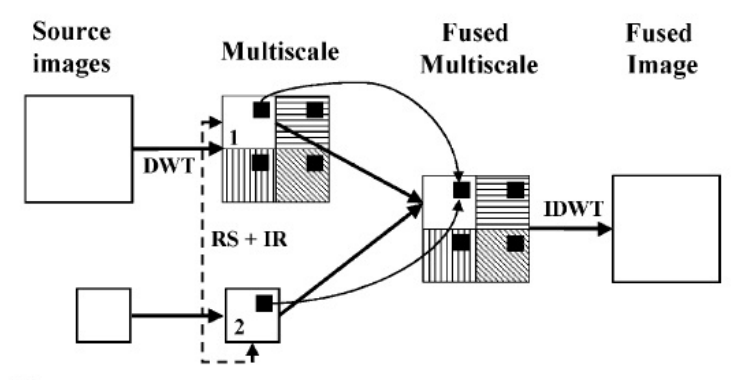

(b)

Fig. 9. Block diagrams of generic fusion schemes where the input images have (a) identical, and (b) different resolutions.

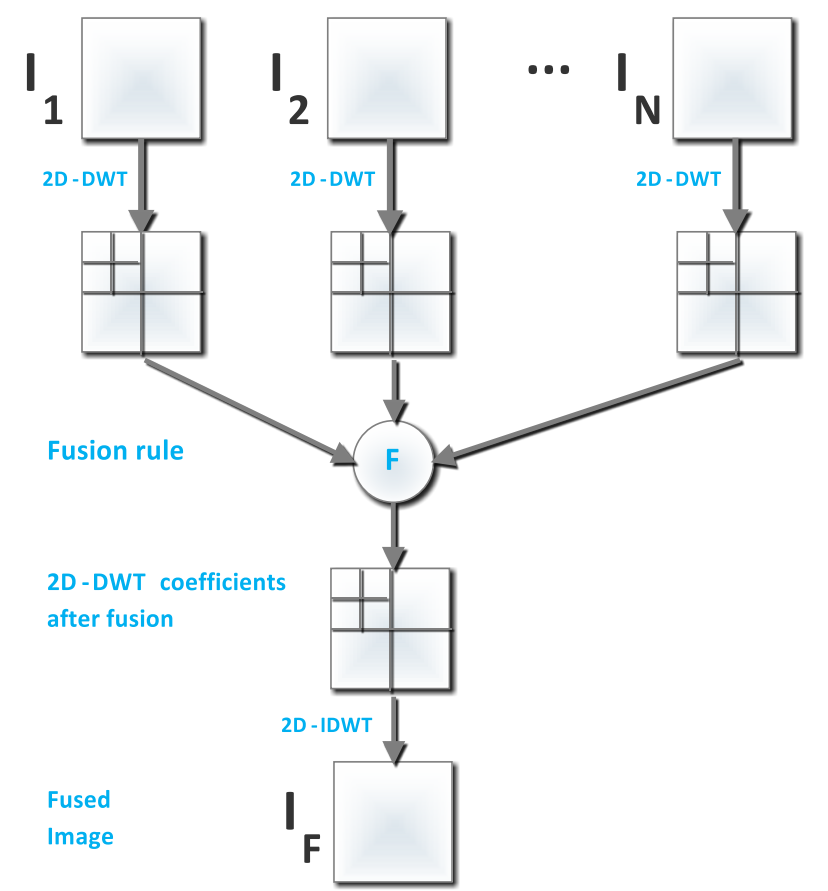

Fig. 10. Image Fusion with the use of 2D-DWT

As far as it concerns the fusion rule or the coefficient selection method, there were tested three different methods, the maximum selection method, the minimum selection method and the mean selection method.

\subsection{Sharpening criteria}

The quality assessment of images can be possible with the use of certain quality measures or criteria. In many cases image sharpening is directly related with the image quality. It is obvious that image details are associated with high frequency components. This means that a high quality image must also have a suitable sharpening level. The criteria used in this work to measure the sharpening level are spatial frequency, entropy, and the Piella and Heijmans metric in cases of image fusion quality evaluation.

Spatial frequency: Spatial frequency of an image $F(i, j)$ with $M x N$ size is defined of the following equations:

$S F=\sqrt{(R F)^{2}+(C F)^{2}}$

with

$R F=\sqrt{\frac{1}{M N} \sum_{m=0}^{M-1} \sum_{n=1}^{N-1}[F(m, n)-F(m, n-1)]^{2}}$

and

$C F=\sqrt{\frac{1}{M N} \sum_{n=0}^{N-1} \sum_{m=1}^{M-1}[F(m, n)-F(m-1, n)]^{2}}$

being the spatial frequencies of rows and columns of an image, respectively. This metric, proposed from $\mathrm{Li}$ et al., [23], measures the total 'activity level' of an image. Thus, it is related with the amount of details contained in an image. Substantially, when the value of spatial frequency grows higher this corresponds to greater image clearness. Consequently, spatial frequency can be used as an evaluation metric of the quality of an image.

Image entropy: Image entropy is given by the following formula:

$$
H=-\sum_{n=0}^{255} p(n) \log _{2} p(n) \text { bits / pixel }
$$

where $p(n)$ is the probability of gray-scale $n$, and it is proportionally related with the amount of information of the image. An image of high sharpening will have a high entropy value.

Piella quality metric: The Piella quality metric was proposed from Piella and Heijmans [27] for the evaluation of quality of image fusion. It derives from the relative metric of Wang and Bovik [25] that also measures the quality of an image. The Wang and Bovik quality index is as follows: assuming $x=\left(x_{1}, x_{2}, \ldots, x_{n}\right), y=\left(y_{1}, y_{2}, \ldots, y_{n}\right)$ are two set of values with the following attributes:

- $\bar{x}$ is the mean value of set $x$,

- $\bar{y}$ is the mean value of set $y$,

- $\sigma_{x}^{2}$ is the variance of set $x$,

- $\sigma_{y}^{2}$ is the variance of set $y$,

- $\sigma_{x y}$ is the covariance of the values from the two sets

The quality index can be expressed as

$Q_{0}=\frac{4 \sigma_{x y} \bar{x} \bar{y}}{\left(\bar{x}^{2}+\bar{y}^{2}\right)+\left(\sigma_{x}^{2}+\sigma_{y}^{2}\right)}$

The above equation can also be written as 
$Q_{0}=\frac{\sigma_{x y}}{\sigma_{x} \sigma_{y}} \frac{2 \bar{x} \bar{y}}{\bar{x}^{2}+\bar{y}^{2}} \frac{2 \sigma_{x} \sigma_{y}}{\sigma_{x}^{2}+\sigma_{y}^{2}}$

The first fraction of $Q_{0}$ constitutes a correlation coefficient between $x$ and $y$. In general, index $Q_{0}=Q_{0}(x, y)$ is a similarity measure between the two vectors, $x$ and $y$ and it varies from -1 to 1 . If the two vectors are replaced from grayscale images then it can become a similarity index of two images. For value $Q_{0}=1$ the two images will be identical, while approaching -1 stands for high unsimilarity of the two images. The second fraction, in the case of images, reflects the distortion of intensity and varies in the interval $[0,1]$. Finally, the third fraction expresses distortion in image contrast between the two images and it also takes values in $[0,1]$.

Piella and Heijmans evolved this measure [26] to use it in image fusion quality assessment. The new measure has the form of $Q(a, b, f)$, with $a$ and $b$ being the two input images and $f$ the fused image. This measure reflects the local coherence of an input image that may depends on local variance in intensity, contrast, clearness or entropy. Considering intensity variance of two input images $a$ and $b$ , a local weight $\lambda$ that indicates the relative significance of image $a$ in comparison with image $b$ is determined. High values of $\lambda$ imply greater importance for image $a$. A typical choice for calculating $\lambda$ is given by the next formula:

$$
\lambda=\frac{S\left(I_{a}\right)}{S\left(I_{a}\right)+S\left(I_{b}\right)}
$$

where $S(I a), S(I b)$ is a measure of salience (i.e. intensity variance) for the two images.

Considering the above, the Piella and Heijmans is formed as:

$$
Q(a, b, f)=\lambda Q_{0}\left(I_{a}, I_{f}\right)+(1-\lambda) Q_{0}\left(I_{b}, I_{f}\right)
$$

Piella and Heijmans proposed that the metric should be calculated locally, using a shifting window. In this case, eq. (2.14) and (2.15) will be transformed as:

$$
\lambda(w)=\frac{S\left(I_{a} / w\right)}{S\left(I_{a} / w\right)+S\left(I_{b} / w\right)}
$$

and

$$
\begin{aligned}
& Q(a, b, f)=\frac{1}{|W|} \sum_{w \in W}\left(\lambda(w) Q_{0}\left(I_{a}, I_{f} / w\right)+\right. \\
& \left.+(1-\lambda(w)) Q_{0}\left(I_{b}, I_{f}(w)\right)\right)
\end{aligned}
$$

Thus, in regions where image $a$ is of higher importance compared to image $b$, the value of index $Q(a, b, f)$ is mainly determined from $a$. Contrariwise, in regions that image $b$ has greater significance to image $a$ will determine index $Q(a, b, f)$ according to image $b$.

\section{Experimental Results and Performance Assessment}

There are two main difficulties concerning the proposed method. First, there is not a single quality measure that can strictly define the sharpening result and second, there are too many parameters that have to be considered during the process.

\section{Experiment 1}

The above mentioned image fusion method has been applied to MR images of human brain acquired with a pulse sequence providing T1-weighted contrast. Processed images were in DICOM format and had a $256 \times 256$ spatial resolution. Initially, a denoising filter that uses the complex 2D dual-tree DWT was applied to an input image as a preprocessing step [33-34]. The performance results based on two sharpening measures, namely Entropy and Spatial Frequency as well as PSNR value, are listed in Table 1. It can be observed that Entropy is improved (increased). Spatial frequency is not affected considerably, whilst contrast is reduced (decreased). The PSNR value is equal to 51.035 .

Table 1. Sharpening metrics from the original MR image and the corresponding image after denoising with complex 2D dual-tree DWT

\begin{tabular}{|lccc|}
\cline { 2 - 4 } & Entropy & $\begin{array}{c}\text { Spatial } \\
\text { Frequency }\end{array}$ & PSNR \\
\hline Input Image & 4.633 & 17.235 & \\
$\begin{array}{l}\text { Denoised } \\
\text { Image }\end{array}$ & 4.7255 & 17.213 & 51.035 \\
\hline
\end{tabular}

Subsequently, five sharpening techniques were independently applied to the denoised image. Afterwards, we applied the above mentioned three metrics to sharpened images and the performance results are illustrated in Table 2.

Table 2. Sharpening measure values of 5 various sharpening techniques for an MR image

\begin{tabular}{cccccc}
\cline { 2 - 6 } & $\begin{array}{c}\text { Unshar } \\
\mathbf{p}\end{array}$ & LCE & $\begin{array}{c}\text { Mask } \\
\text { Filt. }\end{array}$ & $\begin{array}{c}\text { Max- } \\
\text { Min }\end{array}$ & $\begin{array}{c}\text { CLAH } \\
\text { E }\end{array}$ \\
\hline Entropy & 4.626 & 4.757 & 4.988 & 4.944 & 6.691 \\
Spatial & & & & & \\
$\begin{array}{c}\text { Frequenc } \\
\mathbf{y}\end{array}$ & 16.578 & $\begin{array}{c}22.69 \\
0\end{array}$ & $\begin{array}{c}17.14 \\
5\end{array}$ & $\begin{array}{c}28.06 \\
5\end{array}$ & 31.455 \\
\hline
\end{tabular}

For all five sharpening methods employed, the involved parameters were selected according to sharpening measure values and subjective analysis of image quality discussed above. In the sequel, the aforementioned sharpened images were fused using wavelets to achieve better sharpening results. There are various factors which can influence the wavelet-based image fusion results. The core factor in wavelet-based image fusion is the wavelet coefficients combination (i.e. the fusion rule) because it determines how to merge the coefficients in an appropriate way so that a high-quality fused image can be obtained. In this study, three wavelet coefficients combining methods have been used: maximum coefficient rule, minimum coefficient rule and mean value coefficient rule. On a subjective basis, the maximum coefficient rule gave the better fusion results and therefore was chosen to be employed in our experiments. Another critical factor in wavelet-based image fusion is that of the application of a suitable wavelet transform algorithm. We have used three 
different wavelet transforms: the 2D DWT with a decomposition level of 1, the 2D SWT with a decomposition level of 3 and the 2D MDWT, with a decomposition level of 3 . The decomposition level of 3 was selected according to the visual result and the quality performance according to spatial frequency and entropy. Table 3 presents the quality results for a test image in decomposition levels 2 to 7 . A final factor is the selection between the various wavelet functions (e.g. Daubechies's wavelets, Haar wavelets, etc). The sharpened image fusion results associated with the maximum coefficient fusion rule, the Daubechies's 4 (db4) function, along with decomposition level of 1, 3 and 3, as the wavelet-basis for DWT, SWT, and MDWT correspondingly, are presented in Figure 11.

Table 3. Quality measure values for fusion with DWT and SWT in various decomposition levels (for db4 wavelet function)

\begin{tabular}{|ccccc|}
\hline $\begin{array}{l}\text { Decom } \\
\text { position } \\
\text { level }\end{array}$ & $\begin{array}{l}\text { Entrop } \\
\text { y_A } \\
\text { trous }\end{array}$ & $\begin{array}{l}\text { Spatial } \\
\text { Freque } \\
\text { ncy_A } \\
\text { trous }\end{array}$ & $\begin{array}{l}\text { Entr } \\
\text { opy } \\
\text { DW } \\
\text { T }\end{array}$ & $\begin{array}{l}\text { Spatial } \\
\text { Frequen } \\
\text { cy_DWT }\end{array}$ \\
\hline 2 & 4.57 & 16.12 & 4.86 & 17.06 \\
3 & 5.03 & 15.58 & 5.41 & 17.42 \\
\hline 4 & 5.24 & 15.21 & 5.62 & 17.18 \\
5 & 5.36 & 15.23 & 5.90 & 17.14 \\
6 & 5.62 & 15.07 & 6.18 & 17.30 \\
7 & 6.09 & 14.72 & 6.61 & 17.03 \\
\hline
\end{tabular}

To quantitatively evaluate the image quality of the fused images obtained with the three different wavelet algorithms (DWT, SWT and MDWT), three different metrics were employed and the relevant results are displayed in Table 4. As can be observed, the entropy values are improved compared to that of the input image, giving the best performance for fused image obtained by the DWT. The spatial frequency metric is also improved giving the best performance when the DWT is used. The Piella's fusion quality index gives an indication of how much of the salient information contained in each one of the input images has been transferred into the fused image without introducing distortions (i.e. the measure of similarity between fused and input images). The range of its values is $[0,1]$, where the best value of 1 is achieved for the absolutely successful image fusion. We have found that DWT provides the most accurate representation of the original image according to the Piella's fusion quality index.

Table 4. Sharpening index values for the DWT, SWT and MDWT transform algorithms (Db4 wavelet function and maximum coefficient selection were implemented in all cases).

\begin{tabular}{|cccc|}
\cline { 2 - 4 } \multicolumn{1}{c|}{} & Entropy & $\begin{array}{c}\text { Spatial } \\
\text { Frequency }\end{array}$ & $\begin{array}{c}\text { Piella } \\
\text { Metric }\end{array}$ \\
\hline Input Image & 4.633 & 17.235 & - \\
DWT & 6.327 & 21.967 & 0.791 \\
SWT & 6.052 & 20.179 & 0.753 \\
MDWT & 5.998 & 20.217 & 0.656 \\
\hline
\end{tabular}
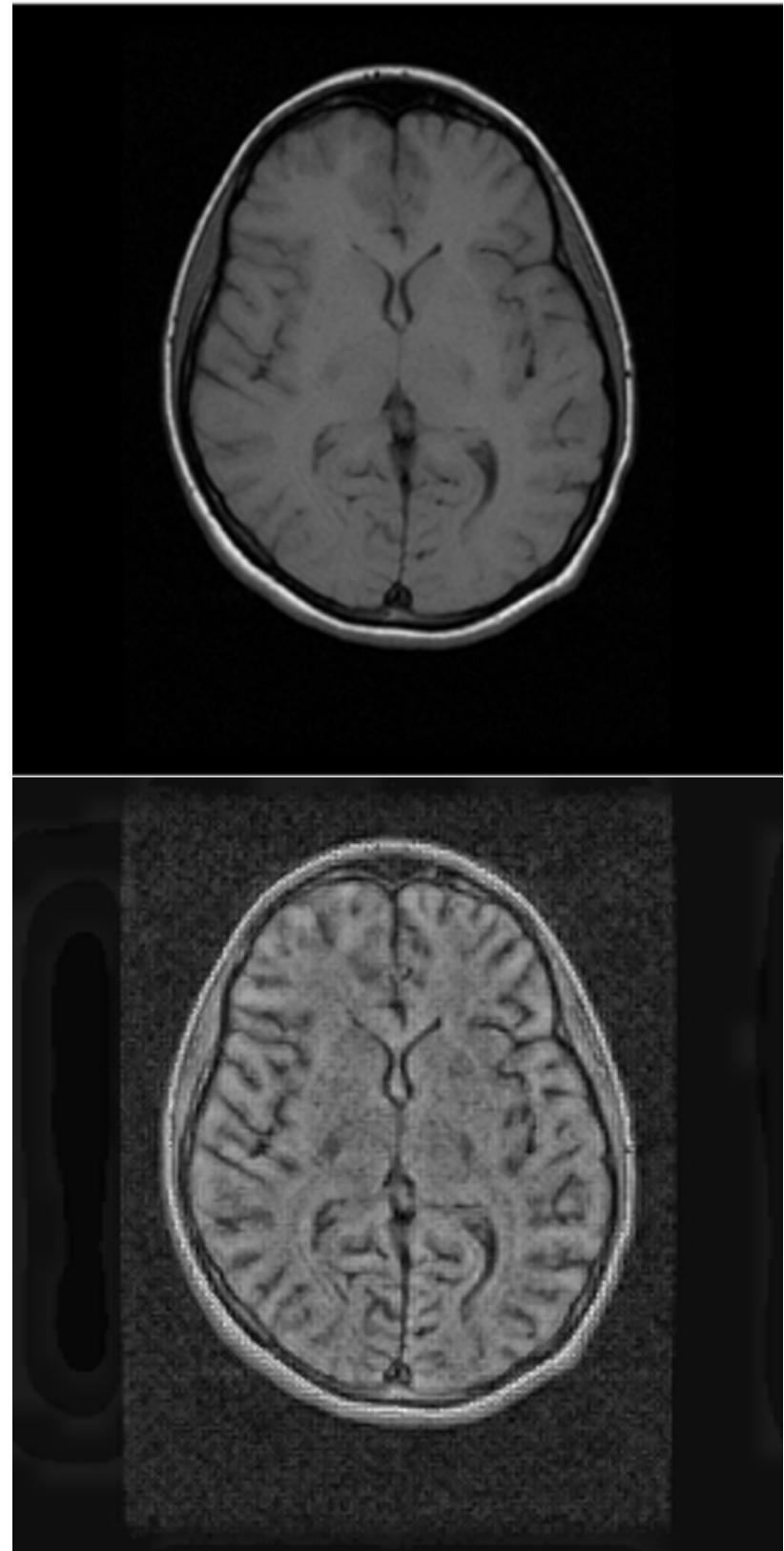

(a) Original Image Fused Image with DWT (max)

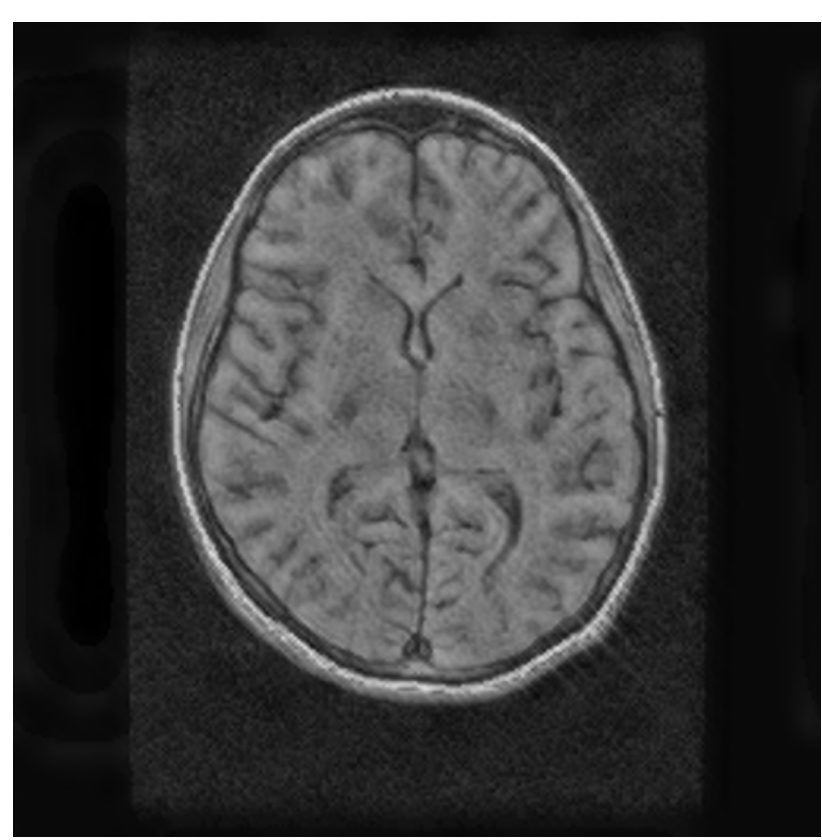




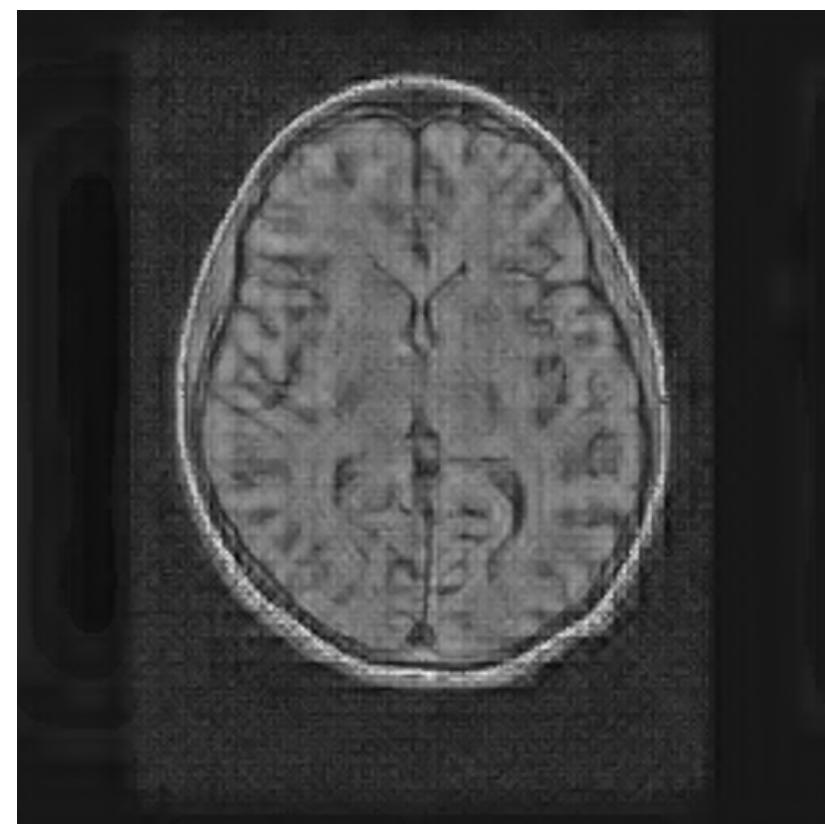
(c)

sed Image with SWT (max) n)Fused Image with MDWT (max) Fig 11. The original MR image (a) and fused images resulting with the use of maximum coefficient rule, the $\mathrm{db} 4$ wavelet function, and three different transform algorithms.

\section{Experiment 2}

In this experiment, we have carried out some comparisons on different wavelet functions of DWT with a decomposition level 1 and by using the maximum coefficient fusion rule. The obtained sharpening measure values of fused image are listed in Table 5. It can be inspected that all wavelet functions gave small fluctuations as regards quality metric values. Thereby, the selection of wavelet function is not considered to be substantial for wavelet-based image fusion. In addition, independent of the wavelet function used, the spatial frequency and entropy are improved to the same extent. Overall, Haar wavelet function seems to perform slightly better than the other functions tested and, therefore, it was adopted for the benchmarking of the proposed approach against other methods (Experiment 4).

Table 5. Sharpening index values for the fused image acquired with various wavelet functions (DWT with decomposition level 1 and the maximum coefficient selection rule were implemented in all cases).

\begin{tabular}{|ccc|}
\cline { 2 - 3 } \multicolumn{1}{c|}{} & Entropy & $\begin{array}{c}\text { Spatial } \\
\text { Frequency }\end{array}$ \\
\hline Input Image & 4.633 & 17.235 \\
Haar & 5.728 & 23.628 \\
Daubechies 4 & 5.827 & 21.967 \\
Biorthogonal 1.3 & 5.813 & 23.379 \\
Rev. Biorthogonal 1.3 & 5.837 & 23.819 \\
Symlets 2 & 5.841 & 23.167 \\
Coiflets 2 & 5.897 & 23.254 \\
Biorthogonal 6.8 & 5.873 & 23.039 \\
Rev. Biortogonal 6.8 & 5.882 & 23.312 \\
\hline
\end{tabular}

\section{Experiment 3}

In order to show the effectiveness of the proposed method, tests were realized on 60 brain MR images. In this experiment, the $\mathrm{db} 4$ function was applied as the wavelet- basis for DWT with a decomposition level of 1, along with the maximum coefficient selection rule. In Figures 12(a) and 12(b) percentage variations of Entropy and Spatial Frequency, respectively, are plotted for the $60 \mathrm{MR}$ images studied. As clearly seen, for a single independent fused image, a greater increase in entropy is accompanied by a greater increase in spatial frequency. In addition, for comparison reasons, average percentage variations of fused image sharpening measures by using $\mathrm{db} 4$ and Biorthogonal 1.3 (bior 1.3) wavelet functions are listed in Table 6.

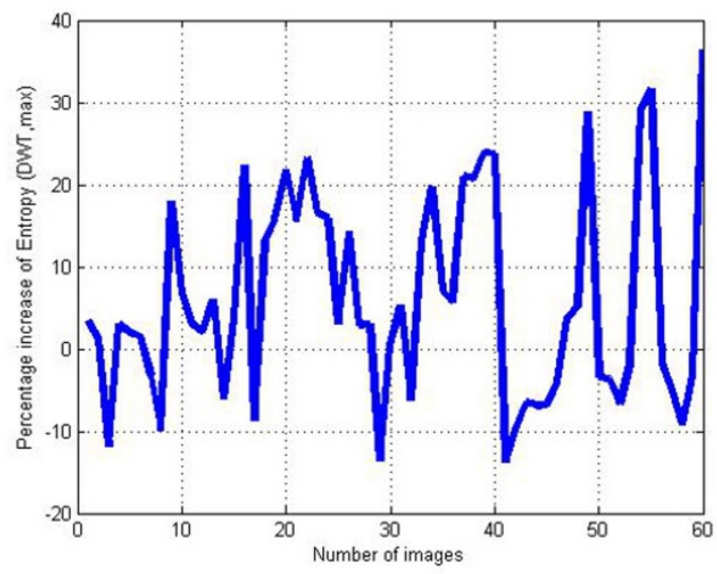

Fig. 12(a. Entropy percentage variation for $60 \mathrm{MR}$ images

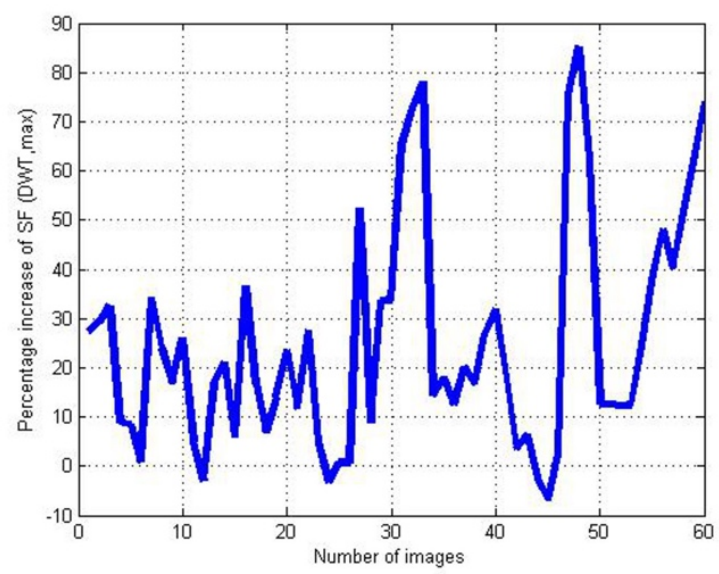

Fig. 12(b): Spatial frequency percentage variation for 60 MR images

Table 6: Average percentage variation of fused image sharpening measures for the $\mathrm{db} 4$ and bior1.3 wavelet functions.

\begin{tabular}{|c|cc|}
\cline { 2 - 3 } & $\begin{array}{c}\text { Average \% Entropy } \\
\text { variation }\end{array}$ & $\begin{array}{c}\text { Average \% } \\
\text { Spatial Frequency } \\
\text { variation }\end{array}$ \\
\hline DB4 & 28.5101 & 54.2035 \\
BIOR 1.3 & 25.5801 & 49.2344 \\
\hline
\end{tabular}

From Table 6, we can inspect that the $\mathrm{db} 4$ wavelet function demonstrates a slightly better performance than bior 1.3 for both Entropy and Spatial frequency measures. 


\section{Experiment 4}

The proposed method was also tested in comparison to two new methods of image sharpening. These methods are described in [35] and [36] respectively. The first technique is a generalised modification of unsharpmasking. The implemetation acquires the regulation of some variables that affect the sharpening result. The variables are: a contrast factor that is a parameter of the adaptive histogram equalization function and the value used is 0.005 , a parameter for enhancing details with the value of 3 used as gain, a binary mask for the filtering process that used the $[0$ $10 ; 111 ; 010$ ] values, a choice for adaptive gain control, a rank of filter that corresponds to median filter and a value of 5 for the iterations of the median filtering.

The other sharpening method is based on wavelets and the right selection of coefficients to eliminate noisy coefficients and keep all the other coefficients of high frequency. There is a comparison between the values of a 3 level decomposition of a DWT with the corresponding coefficients of levels 1 and 2. If their sum is below a threshold value, then the coefficients of the third level are considered as noise and are set to 0 . The application of this technique uses a variable that corresponds to the ratio between the first level threshold $\mathrm{T} 1$ and the second level threshold T2. The default value that was used is 1 . The technique also uses another threshold value which functions similarly to the $k$ parameter of unsharp masking. The value used for these tests was equal to 3. Haar wavelet function is the DWT function used for this method. Haar wavelet function was also used for the proposed method, with one level DWT and maximum wavelet coefficient selection. The output images for the examined techniques are displayed in Figure 13. Table 7 presents the quality measure values estimated for the initial image and the images sharpened with the three techniques.

Table 7. Indices values for the original brain MR image and the corresponding sharpened images shown in Figure 13.

\begin{tabular}{|ccc|}
\hline & Entropy & Spatial Frequency \\
Original image & 4.6334 & 17.2352 \\
DWT(max) & 5.7279 & 23.6284 \\
Deng algorithm & 5.8553 & 40.3971 \\
Ying algorithm & 4.9886 & 32.7688 \\
\hline
\end{tabular}

By analysing the results listed in Table 7 , a consistent trend indicates that the two methods proposed by Deng and Ying individually are superior to the proposed method, mainly regarding the values of spatial frequency, with Deng method giving the best results for the sharpening criteria. Nevertheless, subjective, visual assessment of the corresponding images revealed more artifacts in the images based upon the methods proposed by Deng and Ying compared to those identified in the final fused image resulting from the proposed technique.
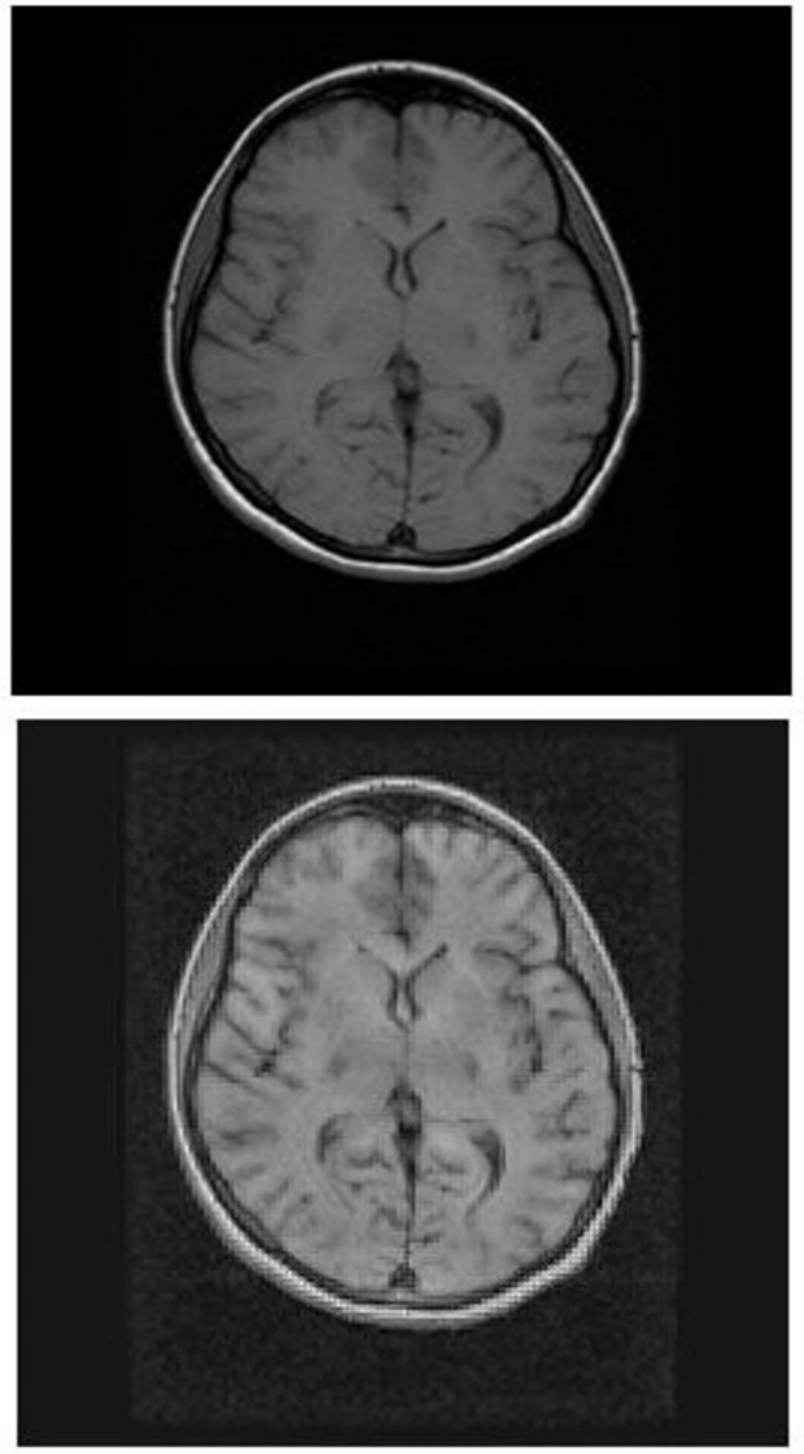

(a) Original Image

Fused Image with DWT(max,Haar)

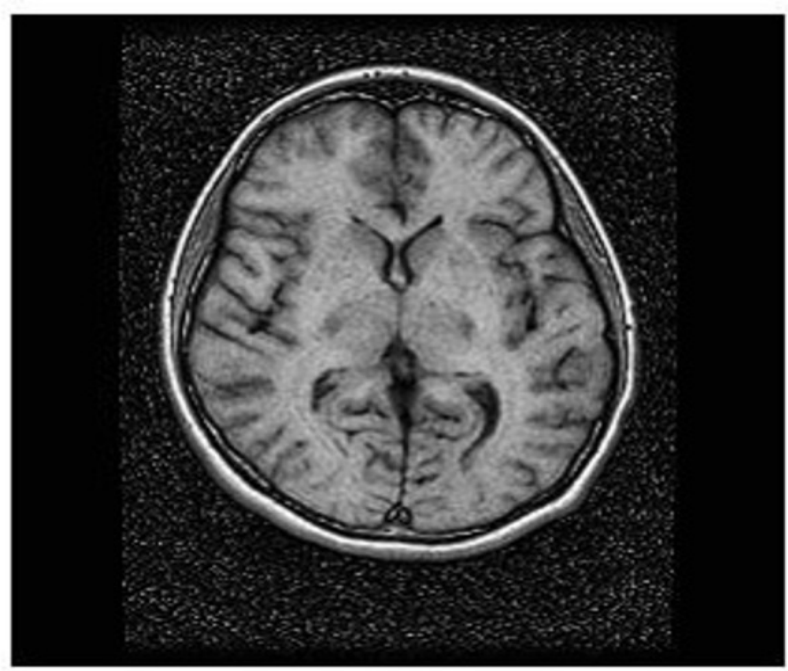




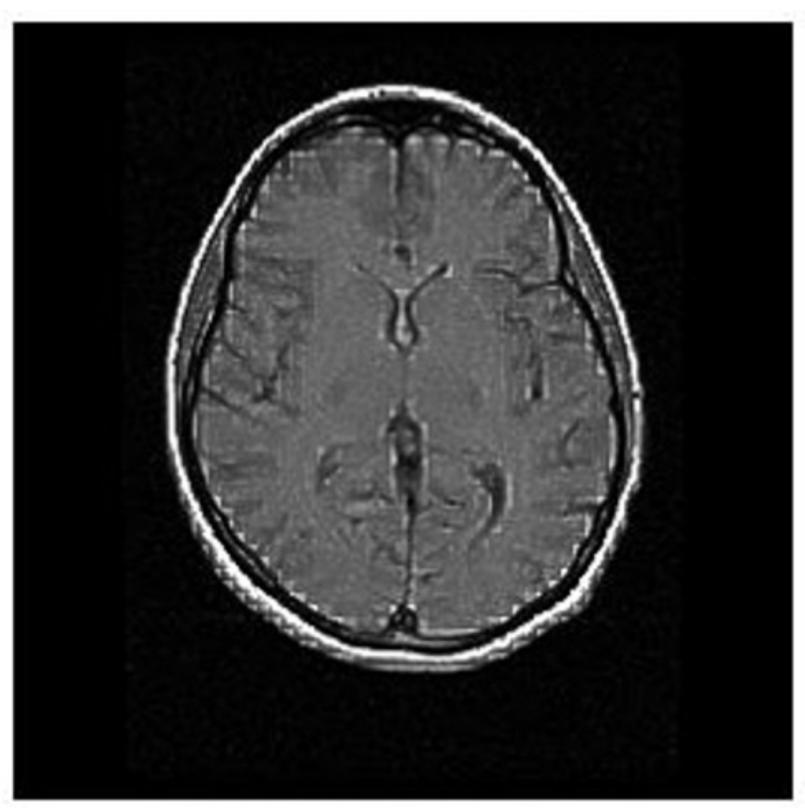

(c) Sharpened Image with the Deng method (d) Sharpened Image with the Ying method

Fig 13. A brain MR image sharpened with 3 different methods: the proposed method with one level DWT fusion with Haar wavelet function and the maximum coefficient selection rule, the Generalized Unsharp Masking Algorithm proposed by Deng and a Wavelet based image sharpening proposed by Ying.

\section{Conclusions}

In this paper we introduce a new method for sharpening of grayscale medical images derived from MR sensors based on multiscale analysis and wavelet fusion. MR images have been selected because of their contrast properties that give relatively poor results when classic sharpening methods are applied to them. The main novelty of the proposed technique lies on that we perform sharpening using only one input image and a multiscale approach that allow as to apply a fusion procedure in different frequency bands of the image. The proposed technique was tested on brain MR images that are usually of low resolution and contrast.

The suggested technique consists of three steps. The first step is denoising, using 2D dual tree Discreet Wavelet Transform in order to remove high frequency noise without affecting the important image details. The second step, which consists of independent sharpening filters that utilize common methods, can be considered as a multiscale approach that emphasizes image details at different frequency subbands of the image. Parameter selection for each of these methods was based on both subjective (i.e., visual comparative evaluation assessing the overall image quality), and objective (i.e., estimation of entropy and spatial frequency in the final image) criteria. The third step comprises the fusion of the 5 resulting images using the 2DDWT method, to further increase the sharpness of the final image. Three different wavelet algorithms were tested: one level of decomposition DWT, the stationary (or undecimated) wavelet transform with 3 decomposition levels, and DWT of 3 decomposition levels. Several wavelet functions as well as 3 different wavelet coefficient selection methods (maximum, minimum, and average value coefficient) were also tested.

The numeric results showed small differences between the values of the sharpening measures in the intercomparison between the various wavelet functions. Furthermore, the maximum coefficient selection method was preferred when choosing the wavelet coefficients because, despite the variations it featured compared to the other two methods tested, it generally performed better in terms of image quality grading.

Moreover, comparison between 1-level DWT, 3-level SWT and 3-level DWT showed that 1-level DWT gave better images in terms of spatial frequency and entropy values. In addition, 1-level DWT exhibits a higher Piella value, which provides a similarity assessment between the individual images used for fusion and the final fused image. Also, 1-level DWT performed better in terms of image quality and artefact identification and, therefore, was preferred over the other two wavelet transforms.

The proposed method was quantitatively applied and tested to 60 brain MR images. The1-level DWT was used in all of them, selecting maximum coefficients for two wavelet functions, namely Daubechies 4 and Biorthogonal 1.3. Daubechies 4 resulted in an increase in values of average entropy $(+28.51 \%)$ and spatial frequency $(+54.2 \%)$. Biorthogonal 1.3 showed average entropy and spatial frequency increases of $25.58 \%$ and $49.23 \%$ respectively. The proposed method was eventually compared to two relatively new image sharpening methods. The first method is an improved generalized application of the unsharp masking algorithm, while the second one is a sharpening technique utilizing DWT and appropriate selection of wavelet coefficients to effectively suppress noise and enhance high frequencies associated with edges. For the proposed method, 1-level DWT with max coefficient selection was used, along with the Haar wavelet function, whereas optimum parameters were chosen for the other two methods. The other two methods outperform the proposed method in terms of spatial frequency values. In subjective analysis, however, the images resulting from the proposed approach present enhanced visual similarity to the initial image, while suppressing artifacts or distortions of basic characteristics/properties, such as the image edges. In any case, the assessment of the efficacy of the proposed methodology in medical imaging requires a rigorous multiparametric procedure which will also take into account diagnostic accuracy.

\section{References}

1. Graps, A., "An introduction to wavelets. IEEE Computational Science and Engineering”, vol. 2, no. 2, pp. 50-61, 1995.

2. Mallat, S.G., "A Wavelet Tour of Signal Processing", second ed. Academic Press, San Diego, 1999.

3. S. G. Mallat, "A theory for multiresolution signal decomposition: the wavelet representation," IEEE Transactions on Pattern Analysis and Machine Intelligence, vol. 11, no. 7, pp. 674-693,1989.
4. S. G. Nikolov, P. Hill, D. R. Bull, and C. N. Canagarajah, "Wavelets for image fusion," in Wavelets in Signal and Image Analysis, Computational Imaging and Vision Series, pp. 213-244, Kluwer Academic Publishers, Dodrecht, The Netherlands, 2001.

5. Shih-Gu Huang, "Wavelet for Image Fusion", National Taiwan University. 
6. R. K. Sharma, M. Pavel, "Adaptive and Statistical Image Fusion", Society for Information Display Digest of Technical Papers, vol. 27, pp. 969-972,1996.

7. A. Garzelli, L. Capobiano and F. Nencini, "Fusion of Multispectral and Panchromatic Images as an Optimization Problem", in Image Fusion - Algorithms and Applications, edited by Tania Stathaki, Academic Press, UK 2008.

8. L. Oudre, Tania Stathaki and N. Mitianoudis, "Image Fusion using optimization of Statistical Measurements", in Image Fusion Algorithms and Applications, edited by Tania Stathaki, Academic Press, UK 2008.

9. V. S. Petrovic and C. S. Xydeas, "Gradient-Based Multiresolution Image Fusion", IEEE Transactions on Image Processing, vol.13, no.2, pp. 228-237, 2004.

10. Y. Yang, D. S. Park, S. Huang, and N. Rao, "Medical Image Fusion via an Effective Wavelet-Based Approach", EURASIP Journal on Advances in Signal Processing, vol. 2010, Article ID 579341, 13 pages.

11. G. Pajares and J-M de la Cruz, "A Wavelet - based Image Fusion Tutorial”, Pattern Recognition, vol.37, pp. 1855-1872, 2004.

12. M. Kumar, P. Ramuhalli, and S. Dass, "A Total Variation Based Algorithm for Pixel Level Image Fusion", IEEE Transactions on Image Processing, September 2009.

13. G. Piella, "A General Framework for Multiresolution Image Fusion: from Pixels to Regions", Information Fusion 4, pp. $259-$ $280,2003$.

14. S.G. Nikolov, Image fusion: a survey of methods, applications, systems and interfaces, Technical Report UoB-SYNERGYTR02,University of Bristol, UK, August 1998.

15. R. K. Sharma, M. Pavel, "Adaptive and Statistical Image Fusion", Society for Information Display Digest of Technical Papers, vol. 27, pp. 969-972,1996.

16. A. Garzelli, L. Capobiano and F. Nencini, "Fusion of Multispectral and Panchromatic Images as an Optimization Problem", in Image Fusion - Algorithms and Applications, edited by Tania Stathaki, Academic Press, UK 2008.

17. T. Fechner, G. Godlewski, "Optimal Fusion of TV and infrared images using artificial neural networks", in: Proceedings of SPIE, vol. 2492, pp. 919 - 925,1995.

18. A. M. Waxman, A. N. Gove, D.A. Fay, J.P. Racamato, J.E. Carrick, M.C. Seibert, E.D. Savoye, "Color night vision: opponent processing in the fusion of visible and IR imagery", Neural Networks, vol.10, no. 1, pp. 1-6, 1997.

19. Z. Xu, "Medical image fusion using multi-level local extrema", Information Fusion, vol. 19, pp. 38-48, Sept. 2014.

20. Zhi-hai $\mathrm{Xu}$, L. Liu, L.Tong, L. Zhou, and C. Chen, "Wavelet medical image fusion algorithm based on local area feature", Biological and Biomedical Reports, vol. 2, no. 1, pp. 25-31, 2012.

21. M. Yasmin, M. Sharif, S. Masood, M. Raza, and S. Mohsin, "Brain Image Enhancement - A Survey”, World Applied Sciences Journal, vol.17, no.9, pp. 1192-1204, 2012.

22. A. Shnayderman, A. Gusev, and A.M. Eskicioglu, "An SVD-Based Grayscale Image Quality Measure for Local and Local Assessment”, IEEE Transactions on Image Processing, vol. 15, no. 2, pp. 422 - 429, February 2006.

23. Shutao Li, James T. Kwok and Yaonan Wang, Combination of Images with Diverse Focuses Using the Spatial Frequency, Information Fusion, Volume 2, Issue 3, Pages 169-176, September 2001.

24. Z. Wang and A. C. Bovik, "A universal image quality index", IEEE Signal Processing Letters, vol. 9, no. 3, pp. 81-84, March 2002.
25. Z. Wang, A. C. Bovik, H.R. Sheikh, and E.P. Simoncelli, "Image quality assessment: from error measurement to structural similarity", IEEE Transactions on Image Processing, vol. 13, no. 4, pp. 600-612, April 2004

26. G. Piella, H. Heijmans, A new quality metric for image fusion, in:International Conference on Image Processing, ICIP, Barcelona, Spain, 2003.

27. W. Shi, C. Zhu, Y. Tian, and J. Nichol, "Wavelet-based image fusion and quality assessment", International Journal of Applied Earth Observation and Geoinformation, vol. 6, pp. 241-251, 2005.

28. W. K. Pratt, Digital Image Processing, Fourth edition, Wiley, USA 2007.

29. R. C. Gonzalez, R. E. Woods, Digital Image Processing, Third Edition, Pearson Education, Inc., USA 2008.

30. M. Sonka, V. Hlavac, R. Boyle, Image Processing, Analysis, and Machine Vision, Brooks/Cole Publishing Company, USA 1999.

31. S. E. Umbaugh, Computer Vision and Image Processing: A Practical Approach Using CVIP tools, Prentice Hall PTR, USA 1998.

32. Liu, Z., Blasch, E., Xue, Z., Langaniere, R., and Wu, W., ("Objective Assessment of Multiresolution Image Fusion Algorithms for Context Enhancement in Night Vision: A Comparative Survey", IEEE Transactions on Pattern Analysis and Machine Intelligence, vol. 34, no. 1, pp. 94-109, 2012.

33. N. G. Kingsbury. Image processing with complex wavelets. Phil. Trans. Royal Society London A, September 1999.

34. N. G. Kingsbury. Complex wavelets for shift invariant analysis and filtering of signals. Applied and Computational Harmonic Analysis, vol. 10, no.3, pp. 234-253, May 2002.

35. G. Deng, "A generalized unsharp masking algorithm" IEEE Transactions Image Processing, vol. 20, pp.1249-1261, 2011.

36. Liu Ying, Ng Tek Ming, Liew Beng Keat, "A Wavelet Based Image Sharpening Algorithm". International Conference on Computer Science and Software Engineering, CSSE 2008, Volume 1: Artificial Intelligence, December 12-14, 2008, Wuhan, China; $01 / 2008$.

37. Jahne, B. and Haubecker, H., "Computer Vision and Applications A guide for Students and Practitioners", Academic Press, 2000.

38. H.P. Kramer and J.B. Bruckner, "Iterations of a nonlinear transformation forenhancement of digital images", Pattern Recognition, vol.7, pp. 53-58, 1975.

39. S. M. Pizer, E. P. Amburn, J. D. Austin, et al.: "Adaptive Histogram Equalization and Its Variations". Computer Vision, Graphics, and Image Processing, vol.39, pp. 355-368, 1987.

40. I. Daubechies. "Ten Lectures On Wavelets". SIAM, 1992.

41. Seul M., O’Gorman L. and Sammon M., Practical Algorithms for Image Analysis, Cambridge University Press, 2001.

42. Ritter, G.X. and Wilson, J.N., Handbook of Computer Vision Algorithms in Image Algebra, CRC Press, 1996.

43. K. Zuiderveld: "Contrast Limited Adaptive Histogram Equalization". In: P. Heckbert: Graphics Gems IV, Academic Press 1994.

44. H. Li, S. Manjunath, S. Mitra, "Multisensor image fusion using the wavelet transform", Graphical Models and Image Processing vol. 57, no. 3, pp. 235-245, 1995.

45. L. Chapman, T. Orr, L. Graham, "Wavelets and image fusion", in: Wavelet Applications in Signal and Image Processing III", vol. 2569, pp. 208-219, 1995.

46. M. Holschneider, R. Kronland-Martinet, J. Morlet and P. Tchamitchian. "A real-time algorithm for signal analysis with the help of the wavelet transform". In Wavelets, Time-Frequency Methods and Phase Space, pp. 289-297. Springer-Verlag, 1989. 